\title{
Multi-Target Robust Waveform Design Based on Harmonic Variance and Mutual Information
}

\author{
Bin Wang $\mathbb{D}^{1}$ and Shuangqi Yu $\mathbb{D}^{2}$ \\ ${ }^{1}$ School of Electrical and Electronic Engineering, Shijiazhuang Tiedao University, China \\ ${ }^{2}$ School of Computer and Communication Engineering, Northeastern University at Qinhuangdao, China \\ Correspondence should be addressed to Bin Wang; wangbinneu@qq.com
}

Received 20 April 2020; Accepted 20 June 2020; Published 3 July 2020

Academic Editor: Remi Léandre

Copyright (C) 2020 Bin Wang and Shuangqi Yu. This is an open access article distributed under the Creative Commons Attribution License, which permits unrestricted use, distribution, and reproduction in any medium, provided the original work is properly cited.

\begin{abstract}
Cognitive radar is an intelligent radar system, and adaptive waveform design is one of the core problems in cognitive radar research. In the previous studies, it is assumed that the prior information of the target is known, and the definition of target spectrum variance has not changed. In this paper, we study on robust waveform design problem in multiple targets scene. We hope that the upper and lower bounds of the uncertainty range of robustness are more close to the actual situation, and establish a finite time random target signal model based on mutual information (MI). On the basis of the optimal transmitted waveform and robust waveform based on MI, we redefine the target spectrum variance as harmonic variance, and propose a novel robust waveform design method based on harmonic variance and MI. We compare its performance with robust waveform based on original variance. Simulation results show that, in the situation of multiple targets, compared to the original variance, the MI lifting rate of robust waveform based on harmonic variance relative to the optimal transmitted waveform in the uncertainty range has great improvement. In certain circumstances, robust waveform based on harmonic variance and MI is more suitable for more targets.
\end{abstract}

\section{Introduction}

The traditional radar's transmitting and receiving methods are usually fixed, which are designed in advance by the radar's tasks and application scenarios, including radar system, waveform parameters, signal processing methods, etc. The fixed radar system and working mode limit the further improvement of radar performance. With the electromagnetic environment becoming more and more complex, the traditional radar technology dealing with interference is not enough. For cognitive radar, by extracting and learning the multi-domain characteristics of the target and environment, it can determine the nature of the interference and identify the interference pattern, so as to take targeted countermeasures. The research of cognitive radar has been widely concerned all over the world.

Adaptive waveform design is one of the core problems in cognitive radar research, and experts and scholars all over the world have done a lot of related work. In [1], an iterative opti- mization method of radar waveform is proposed. This method takes energy and SINR as constraints and performance optimization indexes, respectively. It combines the polarization of radar transmitted waveform with filtering structure for joint robust design. In [2], the authors design the local strongest detector based on relative entropy and the best radar transmitted waveform corresponding to the extended target. In the environment of colored noise, compared with the waveform design method based on mutual information, the waveform design based on relative entropy can significantly improve the detection performance of radar. At the same time, the relationship among the output SNR, mutual information and relative entropy is also discussed. In [3], the interference technology of power constraint is studied. On the basis of the interference technology of minimizing SINR and maximizing mutual information, the minimax robust waveform based on SINR and the minimax robust waveform based on mutual information are proposed, respectively, in the constraint of the uncertain range of the 
target. Both of them are the optimization criteria to maximize the waveform design in the worst case. Although the two optimization criteria are suitable for different situations, their performances are similar in the worst case. In [4], the optimal waveform design of radar detection for extended targets in the specific environment of electronic warfare is studied. In this design method, a waveform optimization method based on SNR with clutter effect is proposed. This method studies the waveform optimization of intelligent radar and silent target, intelligent radar and silent radar, intelligent radar and intelligent target in electronic warfare mode. By optimizing the water-filling method, the waveform design algorithm is further optimized on the basis of minmax. In [5], a new waveform design method of cognitive radar is proposed. The interior point method is used to design the transmitted waveform so as to achieve the best performance of the radar. In [6], the authors focus on the radar multi-target detection. In this paper, a multi-target detection method and an adaptive waveform design algorithm based on information theory are proposed to improve the efficiency of multiple hypothesis detection. This method studies the multi-target detection problem by studying the multiple hypothesis tests. The method is in accordance with the algorithm mentioned above, so as to further improve the detection efficiency. In [7], a new target classification waveform based on spectral variance and information theory is proposed. The advantage of this waveform lies in its strong real-time performance and easy application. The correlation measure of information can be regarded as a range. In this paper, an algorithm is proposed to study the lower bound of the range. This algorithm can greatly improve the accuracy of detection performance. In [8], the authors study the robust waveform of orthogonal frequency division multiplexing, and propose three robust waveform design criteria based on power as waveform optimization criteria, which are considered as useful energy, interference and ignored. By optimizing the orthogonal frequency division multiplexing waveform, the transmitted power constrained by the mutual information of target characteristics and the minimum capacity threshold of the system is minimized, and the transmitted power is studied in the worst case. The research shows that, for any target, the robust waveform will limit the performance in the worst case. In [9], the authors present a robust waveform design method based on mutual information. This method can alleviate the situation that the prior information of radar target cannot meet the parameter estimation, and further guarantee the performance of parameter estimation. In this paper, a hierarchical game model is proposed, and it is found that under this model, robust waveform can better guarantee the performance of parameter estimation. In [10], the authors put forward two hypotheses: no echo in the target and echo in the target. By minimizing the mutual information between the targets to maximize the relative entropy of no echo and echo in the target, the performance of target detection can be improved and the target information can be obtained continuously. This method can not only improve the performance of target detection, but also reduce the transmission error rate. In [11], the authors present a radar waveform design method based on information theory. This method is designed for detecting waveforms of multiple targets. In this design method, the general water-filling waveform is quoted and extended. At the same time, a waveform design criterion based on weighted linear sum of mutual information is redefined. Under the constraints of the new optimization criteria and efficient algorithm, the optimization of waveform is classified into single wave and multiple waves. In [12], the authors present an input signal with arbitrary distribution under the condition of Gaussian noise. The signal is constrained by limited power. In this paper, the mutual information of input and output and the minimum mean square error of the input optimal estimation of given output are taken as reference parameters, and a new calculation formula is defined. The formula solves the derivative of mutual information to SINR, and finds that the result is half of the minimum mean square error. In [13], the impulse response of the target is used to replace the scattering behavior of the target. The waveform design with energy and time as the constraint criteria is studied, and resonance measurement waveform of the target based on information theory is proposed. By comparing the two schemes of optimal target detection and optimal information extraction, the former puts as much energy as possible in the mode of maximum target scattering while the latter distributes energy in the condition of maximizing the mutual information between the target and the radar waveform. In [14], the low resolution wide bistatic angle studied in the previous multi-input and multi-output radar scene is improved to high fidelity bistatic target signal. On this basis, the waveform optimization based on bistatic and single base target signals is studied, respectively, and compared with the situation of nonoptimization, partial optimization of waveform and ignoring bistatic or single base path. In [15], the authors present a method of waveform optimization design for MIMO radar under clutter and colored noise. The method takes low probability of intercept as the criterion of waveform optimization design under mutual information constraints, so as to minimize the total power consumption of the system. This method shows that the total transmitted power is the smallest when the transmitted waveform matches the target, jamming, clutter and noise, and it can effectively improve the performance of radar with low probability of intercept.

In $[1,3,4]$, the authors focus on the signal to interference plus noise ratio when they study the optimization of radar waveform. In $[2,6,7,9-11,13]$, the authors study on the basis of the relevant knowledge of information theory. They focus on maximizing or minimizing mutual information and relative entropy as the criterion of radar waveform optimization. The waveforms designed in $[1,4,8,9]$ are all minmax robust waveforms, and in $[3,8,12]$, the optimization criteria are all power. In [7], the authors mainly study spectral variance and information theory, and also mention the concept of correlation measure range. In [10], the authors put forward two kinds of hypotheses, and use the related knowledge of information theory to realize the target function by minimizing the mutual information of the target and maximizing the relative entropy of the two hypotheses. In [11], the authors study the radar waveform based on information theory. In the research, the authors improve the 


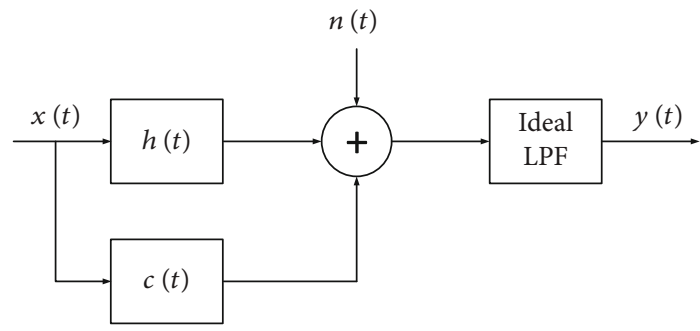

FIgURE 1: Random target signal model for transmitted waveform based on MI.

general water-filling algorithm, classify the single wave and multi wave, and define a new linear weighting formula based on mutual information. In the above articles, where the spectral variance is involved, the original variance formula is used. Most of the research is based on algorithm, research conditions, criteria, environment and other aspects, and no researchers focus on the spectral variance. Or some studies do not consider the actual situation of the target and the robustness of the waveform Therefore, this paper studies the multi-target robust waveform optimization method based on mutual information when the targets are uncertain, that is, the prior probability of the target is unknown. Or some studies only consider single target situation. In this paper, considering the situation of multiple targets, the concept of harmonic mean is introduced, and the variance of target spectrum is redefined, which is recorded as harmonic variance. After introducing harmonic variance, the MI lifting rate of robust waveform based on harmonic variance relative to the optimal transmitted waveform within the uncertainty range has great improvement, and we also associate the lifting rate with the number of targets and other factors, so as to get the relevant conclusions.

\section{Random Target Signal Model}

During the whole working process, radar transmits electromagnetic waves into space, receives the reflected target echo, and extracts relevant information including the target from the echo. In general, we think that the detected targets are random, and their probability of occurrence is uncertain in the radar's work scene. Therefore, we introduce random target signal model based on MI. As shown in Figure 1, the expression is

$$
y(t)=x(t) * h(t)+x(t) * c(t)+n(t)
$$

In fact, the multi-target model is similar to the single target model. The corresponding parameters are the same. The difference is that as the single target expands to multiple targets, the each target energy spectrum variance changes. We suppose that multiple targets are transmitted in the same beam. It is convenient to study all targets as a whole large target.

In this model, $x(t)$ represents the transmitted waveform with limited energy, $h(t)$ represents the impulse response of random target, $c(t)$ represents interference signal, which is Gaussian random process with zero mean, $n(t)$ represents noise, which is also process of zero mean, and $y(t)$ represents

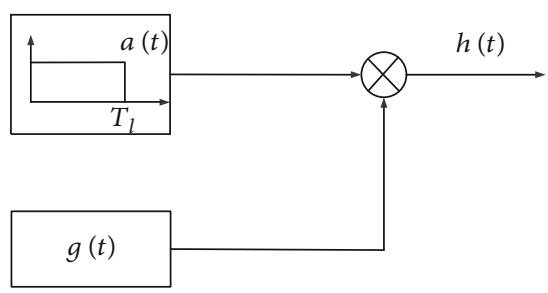

FIGURE 2: Signal model of finite duration for random target.

the output waveform at the output of the low-pass filter. In this random target signal model, we let the duration of the random target be limited. Therefore, we deal with the impulse response to the random target $h(t)$, such as finding a window function with a limited duration and making it perform a certain operation with the impulse response of the random target to obtain a random target impulse response, which is a smooth random process. Figure 2 shows the signal model of random target with finite duration.

In the finite duration signal model of random target, $a(t)$ is function with duration $T_{l}$, and $g(t)$ is generalized stochastic stationary process. The expression of the model is

$$
h(t)=a(t) g(t)
$$

From the expression, we can know that $h(t)$ is the product of $a(t)$ and $g(t)$. So $h(t)$ should also be random stationary process, and it only works on $[0, T l]$. Therefore, the representation of the random target signal model for the transmitted waveform based on MI can be expressed as

$$
y(t)=x(t) *(a(t) g(t))+x(t) * c(t)+n(t)
$$

The process described by this model is process with limited energy, so in this model $x(t)$ and $h(t)$ are energy-limited processes. In the random signal model of finite duration, $h(t)$ is also continuous process. From a mathematical point of view, $h(t)$ is continuously integrable function. Making integration with $h(t)$, the energy constraint of the random signal model of finite duration can be obtained

$$
E_{H}=\int_{T_{l}}|h(t)|^{2} d t=\int_{-\infty}^{\infty}|H(f)|^{2} d f
$$

We define the energy spectral density (ESD) as

$$
\xi_{H}(f)=E\left[|H(f)|^{2}\right]
$$

The energy spectrum variance (ESV) is

$$
\sigma_{H}^{2}(f)=E\left[\left|H(f)-\mu_{H}(f)\right|^{2}\right]
$$

where $\mu_{H}(f)$ is the mean of the random target spectrum, and $H(f)$ is obtained by Fourier transform of $h(t)$, which means $H(f)$ is spectrum response of $h(t)$. 


\section{Optimal Transmitted Waveform Based on MI under Random Target Situation}

We take the MI between the target information and the radar echo as the criterion, and the symbol is expressed as $I(y(t)$; $h(t) \mid x(t))$. The performance of radar detection under the condition of random target is studied, so as to design the relevant optimal transmitted waveform.

As mentioned in equations (1) and (3), $h(t)$ and $g(t)$ are generalized stationary Gaussian random processes. We use the random target signal model for the transmitted waveform based on MI, and substitute (2) into (3), then the target echo output by the low-pass filter can be obtained

$$
y(t)=y_{s}(t)+y_{n}(t)=x(t) * h(t)+x(t) * c(t)+n(t)
$$

where $y_{s}(t)$ represents the signal portion of the target echo, and $y_{n}(t)$ represents the noise interference part of the target echo

$$
\begin{aligned}
& y_{s}(t)=x(t) * h(t) \\
& y_{n}(t)=x(t) * c(t)+n(t)
\end{aligned}
$$

We define that $S_{S}(f)$ is the average power of $y_{s}(t)$ in a finite continuous time, and $S_{N}(f)$ is the average power of $y_{n}(t)$ in a finite continuous time for a Gaussian stochastic process. Mutual information is a measure of the correlation between two event sets. Mutual information is usually represented by joint probability distribution function and edge probability distribution function. In the case of continuous random variables, the mutual information formula can be expressed in the form of integral, so the mutual information between the target information and the radar echo is expressed as

$$
M I=I(y(t) ; g(t) \mid x(t))=T_{l} \int_{B W} \ln \left[1+\frac{S_{S}(f)}{S_{N}(f)}\right] d f
$$

It can be further sorted out that

$$
M I=T_{l} \int_{B W} \ln \left[1+\frac{\sigma_{H}^{2}(f)|X(f)|^{2}}{T_{l}\left\{S_{c c}(f)|X(f)|^{2}+S_{n n}(f)\right\}}\right] d f
$$

In the formula, $S_{c c}(f)$ is the power spectral density (PSD) of the interfering signal, and $S_{n n}(f)$ is the PSD of the noise signal. Detailed derivation can be found in reference [13].

The transmitted waveform based on MI is to optimize the detection performance of the radar system by promoting the mutual information between the target information and the radar echo under certain energy constraints. Therefore, we should maximize the mutual information between target information and the radar echo under certain energy constraints, and then the optimal transmitted waveform based on MI is obtained. The energy constraint is

$$
\int_{B W}|X(f)|^{2} d f \leq E_{H}
$$

We design the optimal transmitted waveform based on MI by maximizing the mutual information between target information and radar echo under certain energy constraints. In other words, we need to find the maximum value of mutual information of independent variables limited by energy constraints. Therefore, we use the Lagrange multiplier method, let (10) be the objective function, and (11) be the constraint condition, then we can obtain

$$
\begin{aligned}
L\left(|X(f)|^{2}, \lambda\right)= & T_{l} \int_{B W} \ln \left[1+\frac{\sigma_{H}^{2}(f)|X(f)|^{2}}{T_{l}\left\{S_{c c}(f)|X(f)|^{2}+S_{n n}(f)\right.}\right] d f \\
& +\lambda\left[E_{H}-\int_{B W}|X(f)|^{2} d f\right]
\end{aligned}
$$

As shown in formula (10), the mutual information between target information and radar echo is a function of $|X(f)|^{2}$. Then, the objective function (12) is also a function of $|X(f)|^{2}$. We can consider that $|X(f)|^{2}$ is the argument of the function, and $L\left(|X(f)|^{2}\right)$ is the dependent variable. We want $L\left(|X(f)|^{2}\right)$ to be the largest, so equation (12) can be written as

$$
\begin{aligned}
L\left(|X(f)|^{2}, \lambda\right)= & T_{l} \int_{B W} \ln \left[1+\frac{\sigma_{H}^{2}(f)|X(f)|^{2}}{T_{l}\left\{S_{c c}(f)|X(f)|^{2}+S_{n n}(f)\right\}}\right] d f \\
& -\lambda \int_{B W}|X(f)|^{2} d f
\end{aligned}
$$

It can be further sorted out that

$$
\begin{aligned}
L\left(|X(f)|^{2}\right)= & T_{l} \ln \left[1+\frac{\sigma_{H}^{2}(f)|X(f)|^{2}}{T_{l}\left\{S_{c c}(f)|X(f)|^{2}+S_{n n}(f)\right\}}\right] \\
& -\lambda|X(f)|^{2}
\end{aligned}
$$

As we want to achieve the goal of maximizing the objective function, mathematically, we achieve this goal by derivation. That is, we solve the derivative of independent variable $L\left(|X(f)|^{2}\right)$ for $|X(f)|^{2}$, and then let its derivative equal to zero, that is

$$
\frac{d L\left(|X(f)|^{2}\right)}{d|X(f)|^{2}}=0
$$

The derivative results can be organized as

$$
\lambda=\frac{S_{n n}(f) \sigma_{H}^{2}(f)}{A(f)|X(f)|^{4}+B(f)|X(f)|^{2}+C(f)}
$$


where $A(f), B(f)$ and $C(f)$ are simplified alternatives

$$
\begin{aligned}
& A(f)=\frac{S_{c c}(f)\left(T_{l} S_{c c}(f)+\sigma_{H}^{2}(f)\right)}{T_{l}} \\
& B(f)=\frac{S_{n n}(f)\left(2 T_{l} S_{c c}(f)+\sigma_{H}^{2}(f)\right)}{T_{l}} \\
& C(f)=\left|S_{n n}(f)\right|^{2}
\end{aligned}
$$

Defining $A=T l / \lambda$, because $\lambda>0$, so the value of $A$ must be positive. Meanwhile, we must ensure that the value of $|X(f)|^{2}$ also be positive, and the expression of $|X(f)|^{2}$ is as follows

$$
|X(f)|^{2}=\max \left[0,-R(f)+\sqrt{R^{2}(f)+S(f)(A-D(f))}\right]
$$

where $D(f), R(f)$ and $S(f)$ are simplified alternatives

$$
\begin{aligned}
& D(f)=\frac{S_{n n}(f)}{\sigma_{H}^{2}(f) T_{l}} \\
& R(f)=\frac{S_{n n}(f)\left(2 T_{l} S_{c c}(f)+\sigma_{H}^{2}(f)\right)}{2 S_{c c}(f)\left(T_{l} S_{c c}(f)+\sigma_{H}^{2}(f)\right)} \\
& S(f)=\frac{S_{n n}(f) \sigma_{H}^{2}(f)}{S_{c c}(f)\left(T_{l} S_{c c}(f)+\sigma_{H}^{2}(f)\right)}
\end{aligned}
$$

For simple calculation, the first-order Taylor approximation is used for calculation.

We define

$$
Q(f)=-R(f)+\sqrt{R^{2}(f)+S(f)(A-D(f))}
$$

Available waveform is

$$
|X(f)|^{2}=\max [0, B(f)(A-D(f))]
$$

where $\bar{B}(f)$ and $\bar{D}(f)$ are simplified alternatives

$$
\begin{aligned}
\bar{B}(f) & =\frac{\sigma_{H}^{2}(f)}{2 T_{l} S_{c c}(f)+\sigma_{H}^{2}(f)} \\
\bar{D}(f) & =\frac{T_{l} S_{n n}(f)}{\sigma_{H}^{2}(f)}
\end{aligned}
$$

$\bar{A}$ is the first order Taylor series of $A$ which is still a constant.

The form after the first-order Taylor series approximation still needs us to solve the value of $|X(f)|^{2}$, and then the mutual information between the target information and the radar echo can be obtained by calculation. We can see that when the value of $S_{c c}(f)$ is 0 , the value of $\bar{B}(f)$ is always 1 . That is to say, when there is no clutter, that is, in the case of pure noise, the clutter factor is 1 .

\section{Robust Waveform Design Based on Harmonic Variance and MI}

In the actual working scene of the radar, most of the target information is not completely known, which leads to the real target cannot be accurately detected. We believe that there is a certain uncertainty range in the real target spectrum. The uncertainty range represents the uncertainty of its target. The larger the uncertainty range is, the greater the uncertainty of the target is. Meanwhile, we believe that there is an optimal transmitted waveform in the uncertainty range, which makes the detection performance of radar system improve as much as possible. If a waveform can be found so that it can get the optimal transmitted waveform in the most unfavorable case of the uncertainty range, then it will be able to make the detection performance of the radar system better than that of the radar system under the optimal transmitted waveform. Therefore, we get harmonic robust transmitted waveform design. Here, we will focus on the multi-target situation.

In the working process of radar, for multi-target scene, because the information of each target is not completely known, the occurrence probability of each target is uncertain. However, the sum of occurrence probability of these targets should be equal to 1 .

Supposing in the multi-target scene, there exists an upper bound and a lower bound in the uncertainty range, and the lower bound is known. Let the uncertainty range be $\varepsilon_{i}$, then

$$
H_{i}(f) \in \varepsilon_{i}=\left\{l_{i k} \leq H_{i}\left(f_{k}\right) \leq u_{i k}, k=1,2 \cdots, k\right\}
$$

where $i=1,2, \cdots, k$ represent different targets, and the uncertainty range of each target is different.

There is an energy constraint in the optimal transmitted waveform design based on MI mentioned above. Similarly, there is also an optimal criterion in the design of the harmonic robust transmitted waveform. The criterion is a function regarding $|X(f)|^{2}$ and $\sigma_{H}^{2}(f)$, and it can be written as $\xi\left(|X(f)|^{2}, \sigma_{H}^{2}(f)\right)$. It should be noted that in the case of single target and multiple targets, $\sigma_{H}^{2}(f)$ is different. Harmonic transmitted waveform design method should meet

$$
\max _{|X(f)|^{2}}\left\{\left.\min _{|H(f)| \in \varepsilon} \xi\left(|X(f)|^{2}, \sigma_{H}^{2}(f)\right)\left|\int_{B W}\right| X(f)\right|^{2} d f \leq E_{H}\right\}
$$

The solution to the maximin optimal problem is

$$
\begin{aligned}
& \left.\xi\left(\left|X^{\max \min }(f)\right|^{2}, \sigma_{H}^{2}(f)\right)\right|_{\int_{B W}\left|X^{\max \min }(f)\right|^{2} d f \leq E_{H}} \\
& \quad \geq\left.\xi\left(\left|X^{\max \min }(f)\right|^{2}, \sigma_{H_{\text {worst }}^{2}}^{2}(f)\right)\right|_{\int_{B W}\left|X^{\max \min }(f)\right|^{2} d f \leq E_{H}} \\
& \quad \geq\left.\xi\left(|X(f)|^{2}, \sigma_{H_{\text {worst }}^{2}}^{2}(f)\right)\right|_{\int_{B W}|X(f)|^{2} d f \leq E_{H}}
\end{aligned}
$$

It can be seen from the above inequality that $\xi\left(|X(f)|^{2}\right.$, $\left.\sigma_{H_{\text {worst }}}^{2}(f)\right)\left.\right|_{\int_{B W}|X(f)|^{2} d f \leq E_{H}}$ represents the optimal transmitted 
waveform in the most unfavorable situation of $H(f)$, that is, in case $H_{\text {worst }}(f)$. The worst target waveform spectrum corresponding to $H_{\text {worst }}(f)$ in the most unfavorable situation in uncertainty range can minimize the objective function output at the end of the receive filter, so that the harmonic robust transmitted waveform in this uncertainty range is the best. Next, we will introduce the MI-based harmonic robust waveform design in multi-target situations.

It can be seen from formula (24) that the harmonic robust transmitted waveform based on MI should meet the following criteria

$$
\max _{|\tilde{X}(f)|^{2}}\left\{\left.\min _{\left|H_{i}(f)\right| \in \varepsilon_{i}} M I\left(|\tilde{X}(f)|^{2}, \sigma_{H}^{2}(f)\right)\right|_{\int_{B W}|\tilde{X}(f)|^{2} d f \leq E_{H}}\right\}
$$

From the above, the expression of MI is

$M I\left(|\tilde{X}(f)|^{2}\right)=T_{l} \int_{B W} \ln \left[1+\frac{\sigma_{H}^{2}(f)|\tilde{X}(f)|^{2}}{T_{l}\left(S_{c c}(f)|\tilde{X}(f)|^{2}+S_{n n}(f)\right)}\right] d f$

We use the Lagrange multiplier method and introduce a Lagrange multiplier to get

$$
\begin{aligned}
L\left(|\tilde{X}(f)|^{2}, \lambda\right)= & T_{l} \int_{B W} \ln \left[1+\frac{\sigma_{H}^{2}(f)|\tilde{X}(f)|^{2}}{T_{l}\left(S_{c c}(f)|\tilde{X}(f)|^{2}+S_{n n}(f)\right)}\right] d f \\
& +\lambda\left[E_{H}-\int_{B W}|\tilde{X}(f)|^{2} d f\right]
\end{aligned}
$$

We will regard $|\tilde{X}(f)|^{2}$ as an argument of a function. If you want to make $L\left(|\tilde{X}(f)|^{2}\right)$ maximum, formula (28) can be written in the following form

$$
\begin{aligned}
L\left(|\tilde{X}(f)|^{2}, \lambda\right)= & T_{l} \int_{B W} \ln \left[1+\frac{\sigma_{H}^{2}(f)|\tilde{X}(f)|^{2}}{T_{l}\left(S_{c c}(f)|\tilde{X}(f)|^{2}+S_{n n}(f)\right)}\right] d f \\
& -\lambda \int_{B W}|\tilde{X}(f)|^{2} d f
\end{aligned}
$$

It can be further sorted out that

$$
\begin{aligned}
L\left(|\tilde{X}(f)|^{2}\right)= & T_{l} \ln \left[1+\frac{\sigma_{H}^{2}(f)|\tilde{X}(f)|^{2}}{T_{l}\left(S_{c c}(f)|\tilde{X}(f)|^{2}+S_{n n}(f)\right)}\right] \\
& -\lambda|\tilde{X}(f)|^{2}
\end{aligned}
$$

Solve the derivative of independent variable $|\tilde{X}(f)|^{2}$ for $L\left(|\tilde{X}(f)|^{2}\right)$, and then its derivative is equal to zero, that is

$$
\frac{d L\left(|\tilde{X}(f)|^{2}\right)}{d|\tilde{X}(f)|^{2}}=0
$$

The derivative results can be organized as

$$
\tilde{\lambda}=\frac{S_{n n}(f) \sigma_{H}^{2}(f)}{\tilde{A}(f)|\tilde{X}(f)|^{4}+\tilde{B}(f)|\tilde{X}(f)|^{2}+\tilde{C}(f)}
$$

where $\tilde{A}(f), \tilde{B}(f)$ and $\tilde{C}(f)$ are simplified alternatives

$$
\begin{aligned}
\tilde{A}(f) & =\frac{S_{c c}(f)\left(T_{l} S_{c c}(f)+\sigma_{H}^{2}(f)\right)}{T_{l}} \\
\tilde{B}(f) & =\frac{S_{n n}(f)\left(2 T_{l} S_{n n}(f)+\sigma_{H}^{2}(f)\right)}{T_{l}} \\
\tilde{C}(f) & =\left|S_{n n}(f)\right|^{2}
\end{aligned}
$$

Defining $\tilde{A}=T_{l} / \tilde{\lambda}$, because $\tilde{\lambda} \geq 0$, so the value of $A$ must be positive. At the same time, we must also guarantee that the value of $|\tilde{X}(f)|^{2}$ be positive. For simplicity of calculation, we introduce a first-order Taylor series, and the expression after first-order Taylor series approximation is as follows

$$
\left|\widehat{X}^{\max \min }(f)\right|^{2}=\max \left[0,-\widehat{R}(f)+\sqrt{\widehat{R}^{2}(f)+\widehat{S}(f)(\widehat{A}-\widehat{D}(f))}\right]
$$

where $\widehat{D}(f), \widehat{R}(f)$ and $\widehat{S}(f)$ are simplified alternatives

$$
\begin{aligned}
& \widehat{D}(f)=\frac{S_{n n}(f)}{T_{l} \sigma_{H}^{2}(f)} \\
& \widehat{R}(f)=\frac{S_{n n}(f)\left(2 T_{l} S_{c c}(f)+\sigma_{H}^{2}(f)\right)}{2 S_{c c}(f)\left(T_{l} S_{c c}(f)+\sigma_{H}^{2}(f)\right)} \\
& \widehat{S}(f)=\frac{S_{n n}(f) \sigma_{H}^{2}(f)}{S_{c c}(f)\left(T_{l} S_{c c}(f)+\sigma_{H}^{2}(f)\right)}
\end{aligned}
$$

Formula (35), (36), and (37) are the results of first-order Taylor approximation. From the above derivation, we can get the right half of inequality (25), that is

$$
\begin{gathered}
\left.M I\left(\left|\tilde{X}^{\max \min }(f)\right|^{2}, \sigma_{H_{\text {worst }}}^{2}(f)\right)\right|_{\int_{B W}\left|\tilde{X}^{\max \min }(f)\right|^{2} d f \leq E_{H}} \\
\quad \geq\left. M I\left(|\tilde{X}(f)|^{2}, \sigma_{H_{\text {worst }}}^{2}(f)\right)\right|_{\int_{B W}|\tilde{X}(f)|^{2} d f \leq E_{H}}
\end{gathered}
$$

We substitute the designed waveform spectrum result into the expression (27) of MI, and perform approximate integral operation on it 


$$
\begin{aligned}
& M I\left(\left|\tilde{X}^{\max \min }(f)\right|^{2}, H(f)\right) \\
& \quad=T_{l} \sum_{k=1}^{k} \Delta f \ln \left[1+\frac{\sigma_{H}^{2}\left(f_{k}\right)\left|\tilde{X}^{\max \min }\left(f_{k}\right)\right|^{2}}{T_{l}\left(S_{c c}\left(f_{k}\right)\left|\tilde{X}^{\max \min }\left(f_{k}\right)\right|^{2}+S_{n n}\left(f_{k}\right)\right)}\right] \\
& \quad=T_{l} \sum_{k=1}^{k} \Delta f \ln \left[1+\frac{\sigma_{H}^{2}\left(f_{k}\right) \cdot \max \left(S_{n n}\left(f_{k}\right), S_{c c}\left(f_{k}\right) \tilde{Q}\left(f_{k}\right)+S_{n n}\left(f_{k}\right)\right)}{T_{l} \cdot \max \left(S_{n n}\left(f_{k}\right), S_{c c}\left(f_{k}\right) \tilde{Q}\left(f_{k}\right)+S_{n n}\left(f_{k}\right)\right)}\right] \\
& \quad \geq T_{l} \sum_{k=1}^{k} \Delta f \ln \left[1+\frac{\sigma_{L}^{2}\left(f_{k}\right) \cdot \max \left(S_{n n}\left(f_{k}\right), S_{c c}\left(f_{k}\right) \tilde{Q}\left(f_{k}\right)+S_{n n}\left(f_{k}\right)\right)}{T_{l} \cdot \max \left(S_{n n}\left(f_{k}\right), S_{c c}\left(f_{k}\right) \tilde{Q}\left(f_{k}\right)+S_{n n}\left(f_{k}\right)\right)}\right] \\
& =M I\left(\left|\tilde{X}^{\max \min }(f)\right|^{2}, H_{\text {worst }}(f)\right)
\end{aligned}
$$

$$
\text { We define } \quad \tilde{Q}\left(f_{k}\right)=-\tilde{R}\left(f_{k}\right)+
$$

$\sqrt{\tilde{R}^{2}\left(f_{k}\right)+\tilde{S}\left(f_{k}\right)\left(\tilde{A}-\tilde{D}\left(f_{k}\right)\right)}$, therefore, $H_{\text {worst }}(f)$ is the most unfavorable spectrum in the uncertain range, which is the worst case. We define the target spectrum variance as

$$
\sigma_{L}^{2}(f)=\frac{1}{M} \sum_{i=1}^{M}\left|P_{i} L_{i}(f)-\frac{M}{\sum_{i=1}^{M}\left(1 / P_{i} L_{i}(f)\right)}\right|^{2}
$$

where $H_{\text {worst }}(f)=L(f), p_{i}$ is the occurrence probability of each target, and $M$ is the target number. Therefore, we can get

$$
\begin{aligned}
& \left.M I\left(\left|\tilde{X}^{\max \min }(f)\right|^{2}, \sigma_{H}^{2}(f)\right)\right|_{\int_{B W}\left|\tilde{X}^{\max \min }(f)\right|^{2} d f \leq E_{H}} \\
& \quad \geq\left. M I\left(\left|\tilde{X}^{\max \min }(f)\right|^{2}, \sigma_{H_{\text {worst }}^{2}}^{2}(f)\right)\right|_{\int_{B W}\left|\tilde{X}^{\max \min }(f)\right|^{2} d f \leq E_{H}}
\end{aligned}
$$

It can be seen that harmonic transmitted waveform design technology considers the transmitted waveform in the worst case, so when designing the waveform, we only consider the lower bound of the uncertainty range. In the uncertainty range, harmonic robust transmitted waveform design concerns that how the transmitted waveform is affected by the uncertainty of the target spectrum. On the basis of guaranteeing the original function of harmonic robust transmitted waveform, the MI-based harmonic robust transmitted waveform design pays more attention to the relative improvement and the relationship with target number of its waveform's MI comparing to that of the optimal waveform in this uncertainty range.

\section{Advantage of Harmonic Variance and Analysis of Performance Parameter}

In the radar working environment, the target captured by the radar system can be either a single target or multiple targets. For multi-target detection, the probability of each target appearing is uncertain.

In actual scenes, the real target cannot be accurately captured, and the robust waveform design uses spectrum estimation to detect the uncertainty range of the target spectrum. The larger the difference of spectrum amplitude between the upper and lower bounds of the uncertainty range is, the greater the uncertainty of the target spectrum is. The real target spectrum changes within this uncertainty range, and robust technology is a good way to guarantee performance in the most unfavorable situations.

The optimal waveform design of the upper and lower bounds of the uncertainty range can greatly improve the performance of the radar system. In the robust waveform design, the lower limit of the target uncertainty range is the most unfavorable target spectrum. Therefore, only the lower bound of the uncertainty range needs to be considered. In other words, we should only consider the most unfavorable conditions. In the robust waveform design, the target spectrum is $H(f)$ and the target energy spectrum variance (ESV) is $\sigma_{H}^{2}(f)$. Since we only consider the situation under the most unfavorable conditions, $|L(f)|$ is the target spectrum under the most unfavorable conditions, then $\sigma_{L}^{2}(f)$ is the ESV in the most unfavorable case, that is $\sigma_{L}^{2}(f)$ is the lower bound of $\sigma_{H}^{2}(f)$. Therefore, our follow-up research will be based on $\sigma_{L}^{2}(f)$.

We take into account that if the robust technology is still applicable when the most unfavorable conditions become more unfavorable, then the uncertainty range applicable to the robust technology can be increased. For the most unfavorable conditions, we only need to consider the $\sigma_{L}^{2}(f)$, that is, reducing the target spectral variance in the most unfavorable situation. 


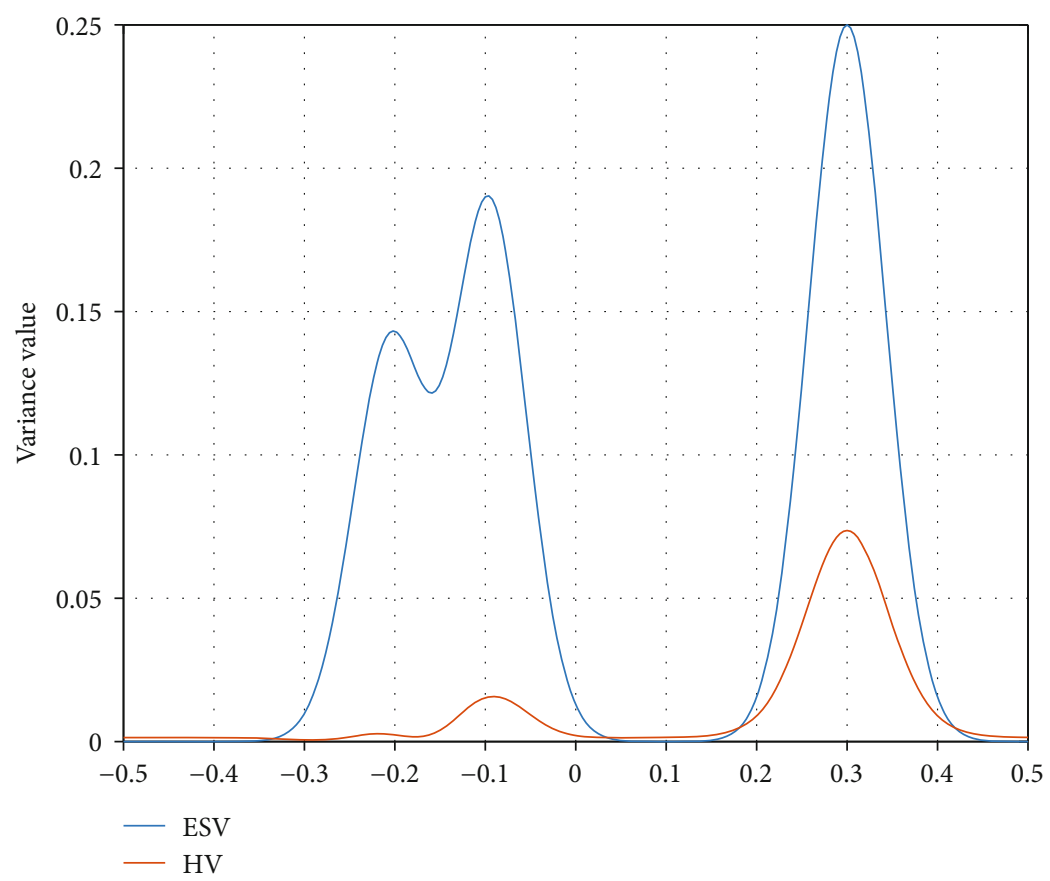

Figure 3: Comparison of the numerical values of the original target ESV and HV.

In mathematics, averages are divided into three categories: arithmetic averages, harmonic averages, and geometric averages. Among these averages, geometric averages are mainly used to calculate the average development rate, while the other two averages are relatively broad usage. Therefore, we consider and compare the arithmetic average and the harmonic average. An example is as follows: suppose that there are two students A and B who participate in the selection of the competition, and finally determine the candidate to pass the selection based on the average of the two students' results in each project. The scores for A are 40, 86, 79, 78, 84, 81, 92, and the scores for B are $56,76,72,83,80,81,78$, respectively.

If calculated with the arithmetic mean, then

$$
\begin{aligned}
& \bar{X}_{A}=\frac{1}{n} \sum X=77.1429 \\
& \bar{X}_{B}=\frac{1}{n} \sum X=75.1429
\end{aligned}
$$

The result is admission A.

If the harmonic mean is used, then

$$
\begin{aligned}
& \bar{X}_{A}=\frac{n}{\sum 1 / n}=71.3567 \\
& \bar{X}_{B}=\frac{n}{\sum 1 / n}=73.9815
\end{aligned}
$$

The result is admission B.

It can be seen from above that $\mathrm{A}$ is only due to the achievement of a project with a score of 40 , which leads to a relatively small harmonic mean. It means that the harmonic mean pays more attention to the influence of smaller values. According to the variance standard formula and the har- monic mean formula, we introduce a new the target spectral variance. In order to facilitate the distinction, the new target spectral variance will be called the harmonic target spectral variance (HESV), referred to as the harmonic variance (HV).

Let the HESV be $\sigma_{L}^{2}(f)$ and its expression is

$$
\sigma_{L}^{2}(f)=\frac{1}{M} \sum_{i=1}^{M}\left|P_{i} L_{i}(f)-\frac{M}{\sum_{i=1}^{M}\left(1 / P_{i} L_{i}(f)\right)}\right|^{2}
$$

The expression of the original target ESV is

$$
\sigma_{H}^{2}(f)=\sum_{i=1}^{M} P_{i}\left|H_{i}(f)\right|^{2}-\left|\sum_{i=1}^{M} P_{i} H_{i}(f)\right|^{2}
$$

We simulated the original target ESV and HV under exactly the same conditions. As can be seen from Figure 3, the HV value is much smaller than the original ESV value. It means that the harmonic variance can well meet our needs to reduce the lower bound of the uncertainty range.

At the same time, in Figures 4 and 5, we simulate the fuzzy model and waveform spectrum results based on harmonic variance for multiple targets. Taking three targets as an example, Figure 4 shows the uncertain range of three targets. During the range, the upper bound of the uncertainty range is composed of the spectrum of the real target plus a random number, and the lower bound is composed of the spectrum of the real target minus a random number. Figure 5 shows the waveform spectrum of the three targets based on the harmonic variance results. In Figure 5, the first small graph shows the spectrum of the real target ESV, the most unfavorable target ESV, and the interference signal of the three targets. The second small graph shows 


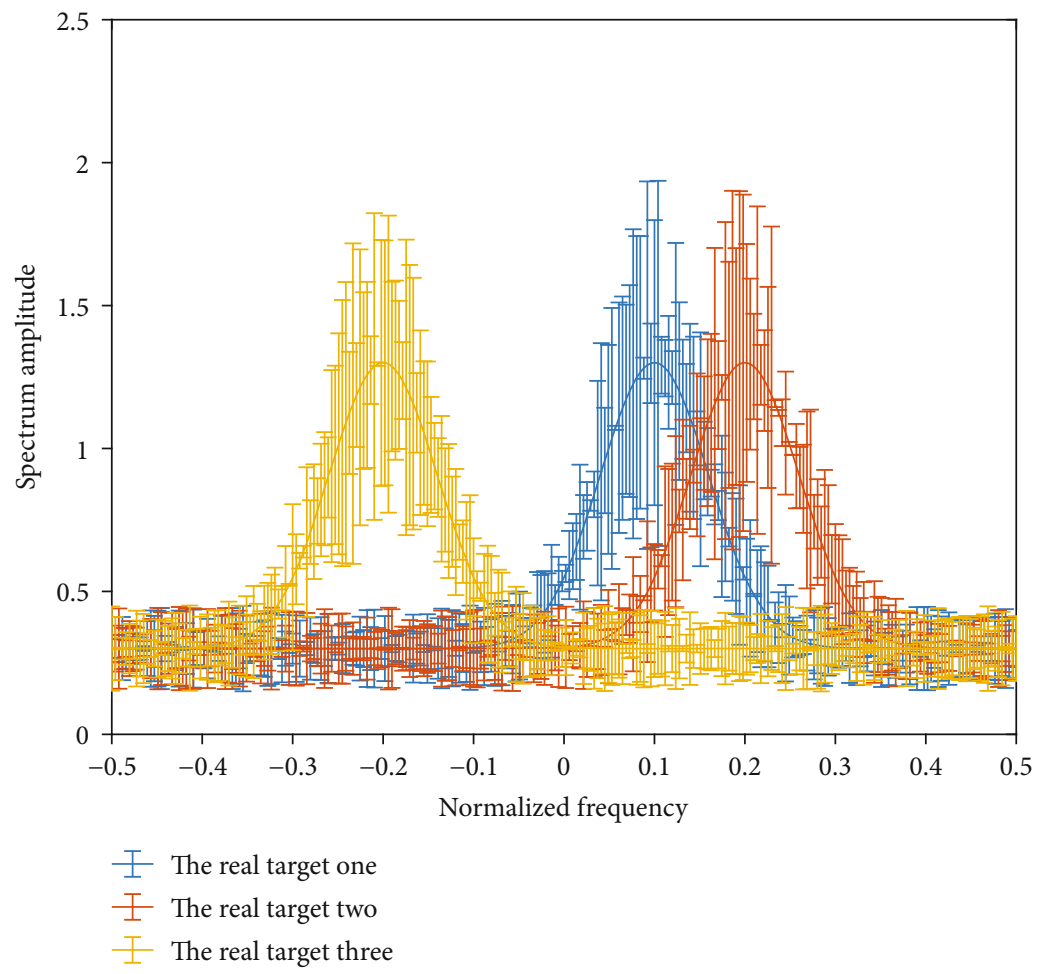

Figure 4: Fuzzy model of multiple targets.

the optimal transmitted waveform spectrum and the harmonic robust spectrum based on $\mathrm{MI}$ in the uncertainty range. In Figure 5, we can see that the main energy is allocated around the sampling frequency with a normalized frequency of -0.2 and 0.1 , respectively, and the designed optimal transmitted waveform spectrum and the robust waveform spectrum based on MI in the uncertainty range are the same as and the real target spectrum. That is to say, after replacing the original variance with the harmonic variance, the robust waveform can still achieve its original features.

In robust waveform design, we concern how the transmitted waveform designed based on MI is affected by the uncertainty of the target spectrum. The design process in the most unfavorable situation, that is, the lower bound of the uncertainty range, can ensure the performance of the radar system and design the robust waveform. After modifying the variance in the robust waveform to the harmonic variance, the part we pay more attention to is the MI lifting rate of robust waveform based on harmonic variance relative to the optimal transmitted waveform in the uncertainty range. Let the relative lifting rate be $\eta$, the MI of robust waveform based on harmonic variance be $M I_{H E S V}$, and the MI of the optimal transmitted waveform in the uncertainty range be $M I_{E S V}$. We can obtain the formula for the lift rate

$$
\eta=\frac{M I_{H E S V}-M I_{E S V}}{M I_{E S V}}
$$

The improvement of the lifting rate means that the MI of the harmonic robust waveform is improved more than that of the optimal transmitted waveform in the uncertainty range, which means that the performance improvement of the harmonic robust waveform is better than the optimal transmitted waveform in the uncertainty range.

\section{Simulation Results and Analysis}

In order to prove our ideas more intuitively, we conducted related simulation experiments. In the simulation experiment, we set the number of sampling points to 256 and the sampling interval to $1 / 256$, and we assume that the interference waveform, noise waveform, and target energy spectrum variance are all known. It is known that the occurrence frequency of the target is less than 1 and is a positive value. At the same time, the total appearance probabilities of the targets should be 1 .

6.1. Comparative Analysis of MI Lifting Rate Based on Original Target Spectrum Variance and Harmonic Variance. In order to compare the lifting rate situation of original target spectrum variance and harmonic variance, we conduct a lot of simulations and analyze them. The following six Figures 68 show the comparison of MI performance based on original variance and harmonic variance with three, four and five targets, respectively. We know that MI can be used to represent the detection performance of the radar system. The figures clearly show that the MI of the robust waveform based on harmonic variance is higher than that of the optimal transmitted waveform in the uncertainty range. That is to say, the performance of the robust waveform based on harmonic variance is better than that of the optimal transmitted waveform in the uncertain range. 

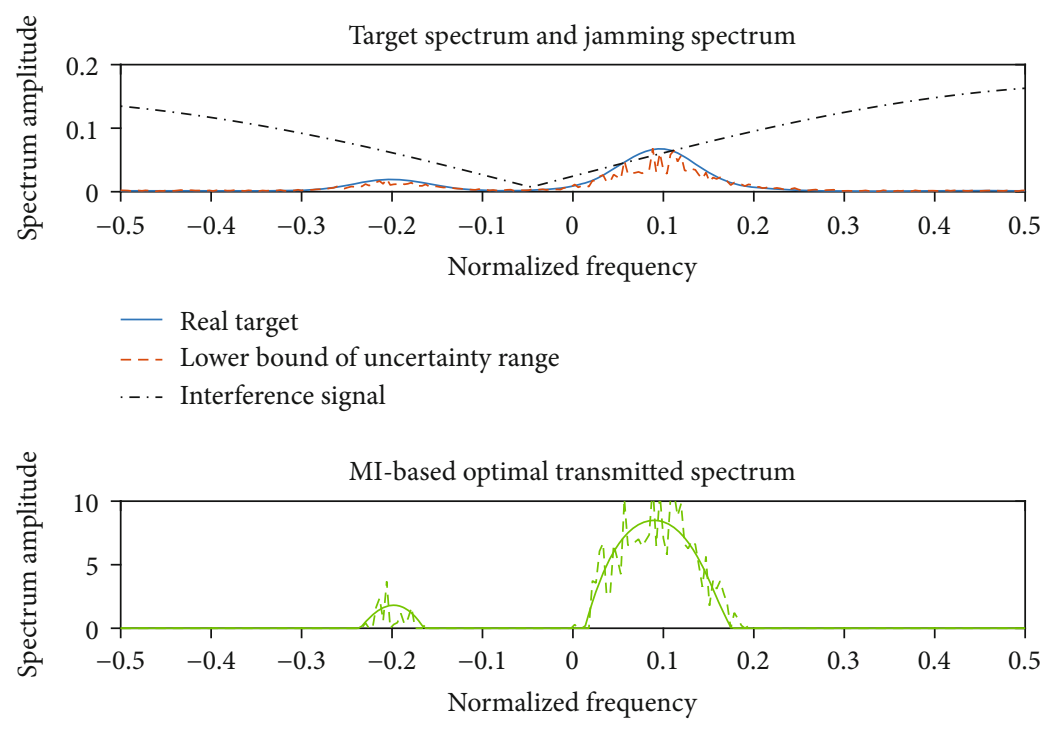

- Optimal transmitted waveform

Robust transmitted waveform

FIGURE 5: Waveform spectrum results of multiple targets.

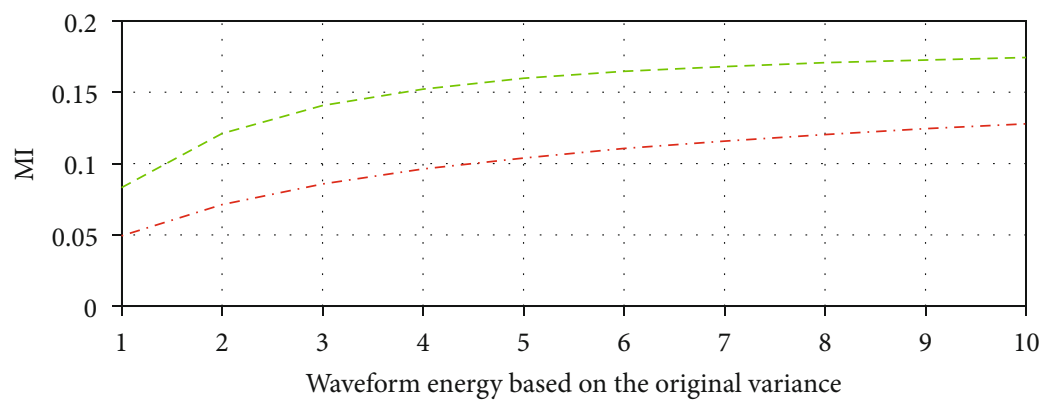

...- Optimal waveform for MI at its worst case

- - Robust waveform for MI at its worst case

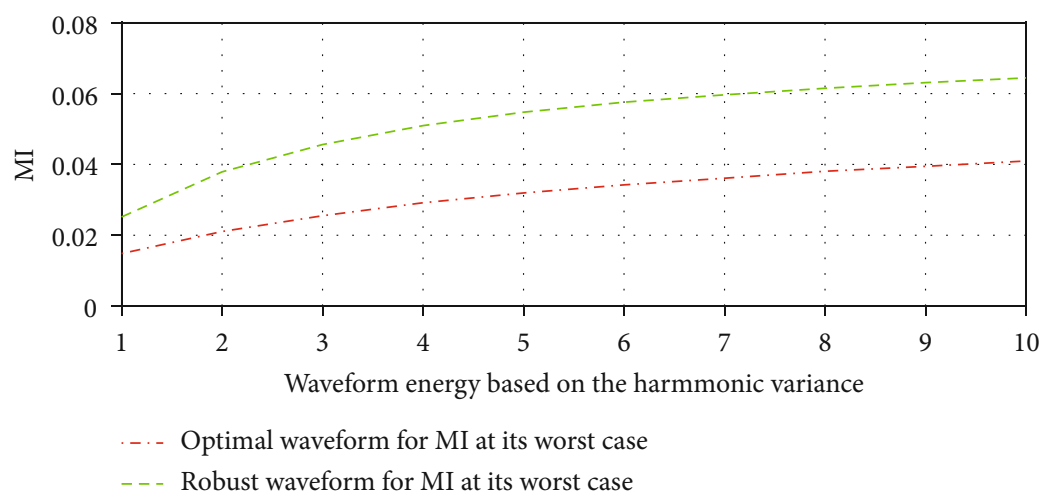

FIGURE 6: MI comparison of three targets based on the original variance and harmonic variance.

After determining that the designed robust waveform based on harmonic variance can still achieve the effect of the original variance, we aim at MI lifting rate with harmonic variance to research. According to the data in Figure 6 to Figure 8, we calculated the relative lifting rate $\eta$. As shown in Tables 1-3, RESV (Relative lifting rate of ESV) in the table is the relative lifting rate based on the original target spectrum variance, and RHESV (Relative lifting rate of HESV) is the relative lifting rate based on the harmonic variance. In order to facilitate observation, we integrated the calculated relative lifting rates of the target numbers three, four, and five into one graph (Figure 9). The first small figure is the relative 


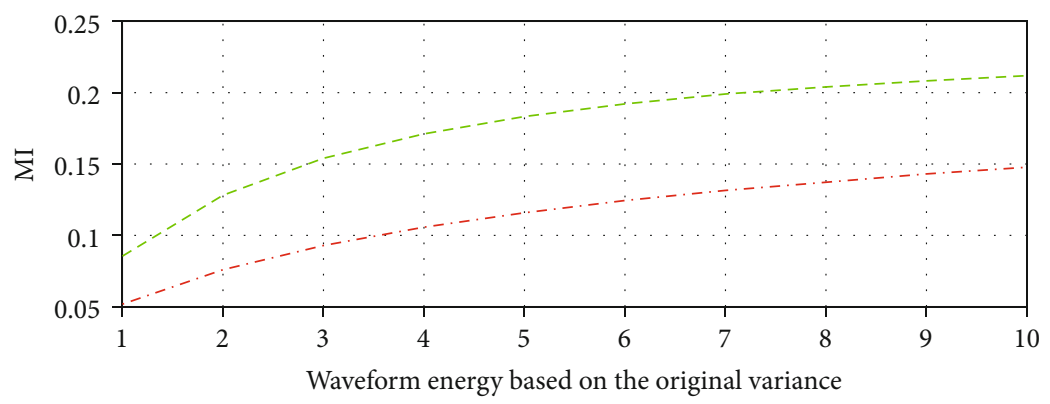

. . - Optimal waveform for MI at its worst case

- - Robust waveform for MI at its worst case

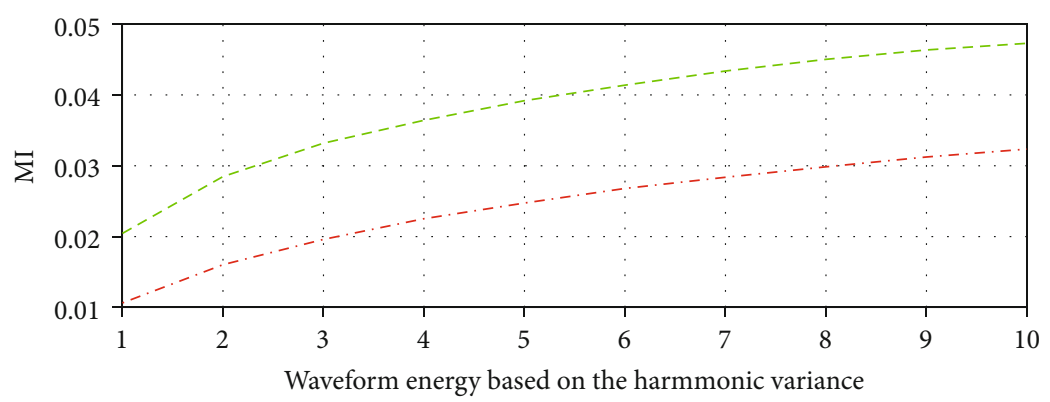

. - Optimal waveform for MI at its worst case

- . Robust waveform for MI at its worst case

FIGURE 7: MI comparison of four targets based on original variance and harmonic variance.

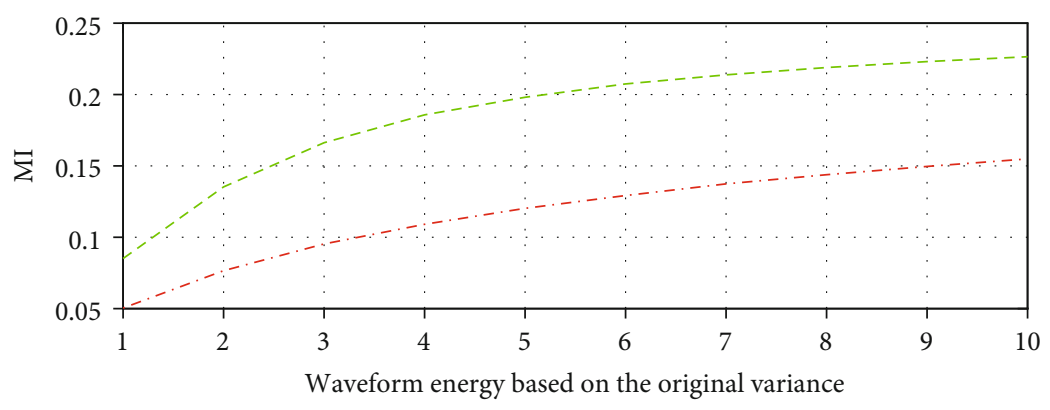

... Optimal waveform for MI at its worst case

- - Robust waveform for MI at its worst case

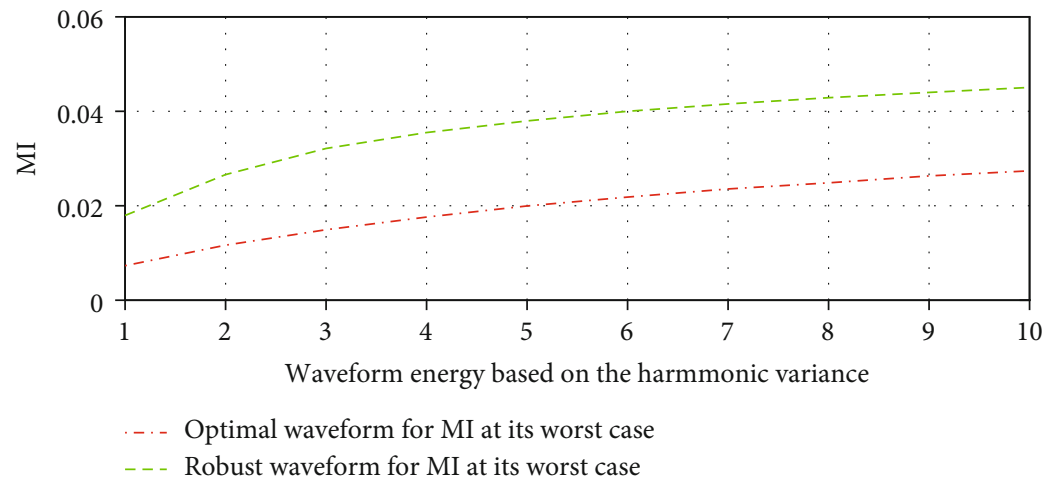

FIGURE 8: MI comparison of five targets based on original variance and harmonic variance.

lifting rate of the harmonic variance and the original variance in the situation of three targets, and the second small figure and the third small figure are four targets and five targets, respectively. We can clearly see that under the same conditions, RHESV is higher than RESV, no matter what the number the target is. 
TABLE 1: The relative lifting rate of three targets.

\begin{tabular}{lcccccccccc}
\hline & 1 & 2 & 3 & 4 & 5 & 6 & 7 & 8 & 9 & 10 \\
\hline RESV & 0.6000 & 0.7143 & 0.6471 & 0.5957 & 0.5490 & 0.4727 & 0.4561 & 0.4166 & 0.3870 & 0.3438 \\
RHESV & 0.7333 & 0.7272 & 0.6981 & 0.7000 & 0.6667 & 0.6429 & 0.6216 & 0.5897 & 0.6000 & 0.5663 \\
\hline
\end{tabular}

TABLE 2: The relative lifting rate of four targets.

\begin{tabular}{lccccccccrrr}
\hline & 1 & 2 & 3 & 4 & 5 & 6 & 7 & 8 & 9 & 10 \\
\hline RESV & 0.6667 & 0.7067 & 0.6522 & 0.6038 & 0.5546 & 0.5600 & 0.5185 & 0.5000 & 0.4828 & 0.4533 \\
RHESV & 0.9048 & 0.7742 & 0.7368 & 0.7045 & 0.6667 & 0.5849 & 0.5714 & 0.5333 & 0.4921 & 0.4769 \\
\hline
\end{tabular}

TABLe 3: The relative lifting rate of five targets.

\begin{tabular}{lcccccccrrr}
\hline & 1 & 2 & 3 & 4 & 5 & 6 & 7 & 8 & 9 & 10 \\
\hline RESV & 0.6364 & 0.7500 & 0.7500 & 0.6957 & 0.6800 & 0.5926 & 0.5634 & 0.5333 & 0.4839 & 0.4688 \\
RHESV & 1.5714 & 1.3043 & 1.1333 & 1.0294 & 0.9000 & 0.8605 & 0.8174 & 0.7200 & 0.6731 & 0.6296 \\
\hline
\end{tabular}
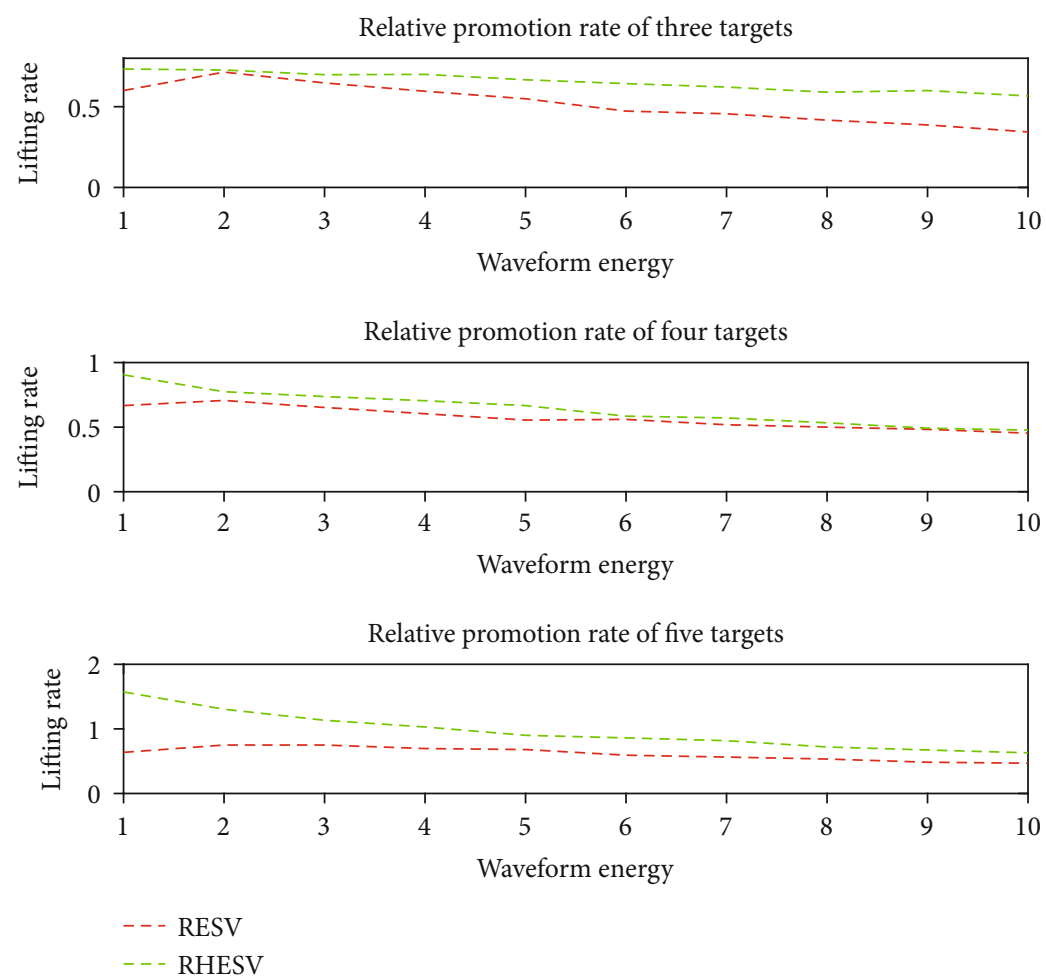

FIGURE 9: Comparison of relative lifting rate.

6.2. Comparative Analysis of MI Lifting Rate and the Number of Targets Based on Harmonic Variance under the Condition of Unequal Probability of Targets. For multi-target scenarios, there are multiple targets in the radar launch environment that need to be detected, and the occurrence probability of each target among all the targets is uncertain. In most cases, the occurrence probability of each target is not exactly the same, but the total occurrence probability of these targets is 1. Next, we will study the case in which the occurrence probability of each target is not equal.
We take the target with the number three, four, five, six, seven, randomly determine their occurrence probability, and make the total probability of the targets be 1 . We conduct a simulation analysis, and obtain the MI performance comparison between the original variance and harmonic variance with the number of targets three, four, five, six, seven. Then we further calculate the MI lifting rate of robust waveform based on harmonic variance relative to the optimal transmitted waveform in the uncertainty range when the target number is three, four, five, six, seven. Table 4 shows the MI lifting 
TABLE 4: MI lifting rate of robust waveform based on harmonic variance relative to the optimal transmitted waveform in the uncertainty range with different target numbers.

\begin{tabular}{lcccccccccc}
\hline & 1 & 2 & 3 & 4 & 5 & 6 & 7 & 8 & 9 \\
\hline Three targets & 0.7333 & 0.7272 & 0.6981 & 0.7000 & 0.6667 & 0.6429 & 0.6216 & 0.5897 & 0.6000 & 0.5663 \\
Four targets & 0.9048 & 0.7742 & 0.7368 & 0.7045 & 0.6667 & 0.5849 & 0.5714 & 0.5333 & 0.4921 & 0.4769 \\
Five targets & 1.5714 & 1.3043 & 1.1333 & 1.0294 & 0.9000 & 0.8605 & 0.8174 & 0.7200 & 0.6731 & 0.6296 \\
Six targets & 2.0000 & 1.8750 & 1.3333 & 1.2857 & 1.0000 & 1.0000 & 0.9474 & 0.8750 & 0.7273 & 0.7333 \\
Seven targets & 2.1111 & 1.9067 & 1.5500 & 1.3333 & 1.2222 & 1.0667 & 0.9394 & 0.8857 & 0.8108 & 0.7436 \\
\hline
\end{tabular}

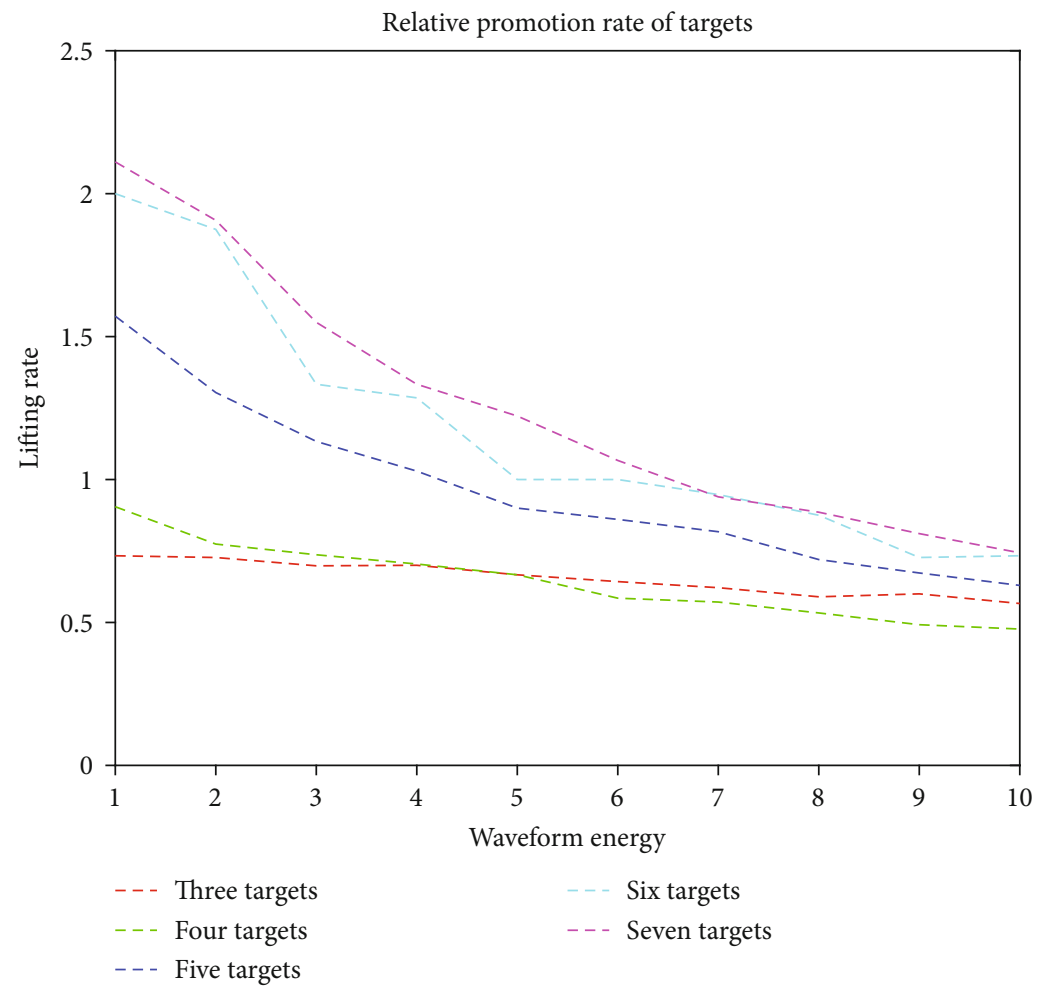

FIGURE 10: MI lifting rate of robust waveform based on harmonic variance relative to the optimal transmitted waveform in the uncertainty range with different target numbers.

rate of robust waveform based on harmonic variance relative to the optimal transmitted waveform in the uncertainty range with different target numbers.

In order to facilitate observation and analysis, we put MI lifting rate $\eta$ with target number three, four, five, six, seven in a graph for comparison, as shown in Figure 10. From the figure, we can intuitively see that as the number of targets increases, MI lifting rate of robust waveform based on harmonic variance relative to the optimal transmitted waveform in the uncertainty range also increases. That is to say, under the same conditions, the greater the number of targets is, the more the MI lifting rate of robust waveform based on harmonic variance relative to the optimal transmitted waveform in the uncertainty range is. Meanwhile the radar performance improves more. In short, under the same conditions as the optimal transmitted waveform in the uncertain range, the more the number of targets is, the better performance the robust waveform based on harmonic variance is.
6.3. Comparative Analysis of MI Lifting Rate and the Number of Targets Based on Harmonic Variance under the Condition of Equal Probability of Targets. We can determine that in the case of multiple targets, the occurrence probability of each target is uncertain. In most cases, the occurrence probability of each target is not the same, but the total occurrence probability is 1 . However, in many cases of unequal probability, the case of equal probability of all targets can be regarded as a special case of unequal probability. Then, we also distinguish the case of equal probability of all targets from the case of unequal probability, and consider the research separately.

Figures 11 and 12 show the relationship between the relative lifting rate and the number of targets in the situation of unequal probability and equal probability target when adopting harmonic variance. In the figure, the abscissa is the target number, and the ordinate is the relative lifting rate, and each line represents its waveform energy distribution is exactly the same. As can be seen in Figure 11, the line starts to rise 


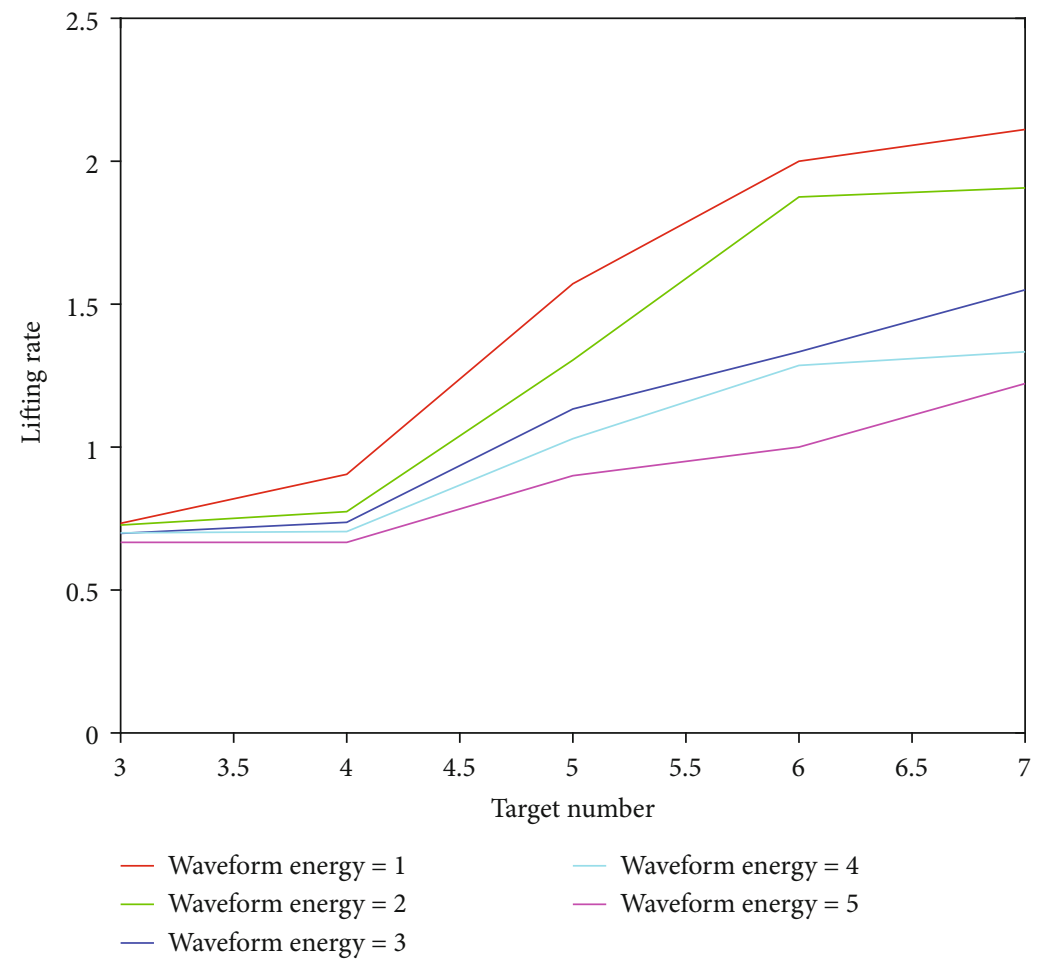

FIGURE 11: The relationship between the relative lifting rate and the number of targets in the situation of unequal probability target.

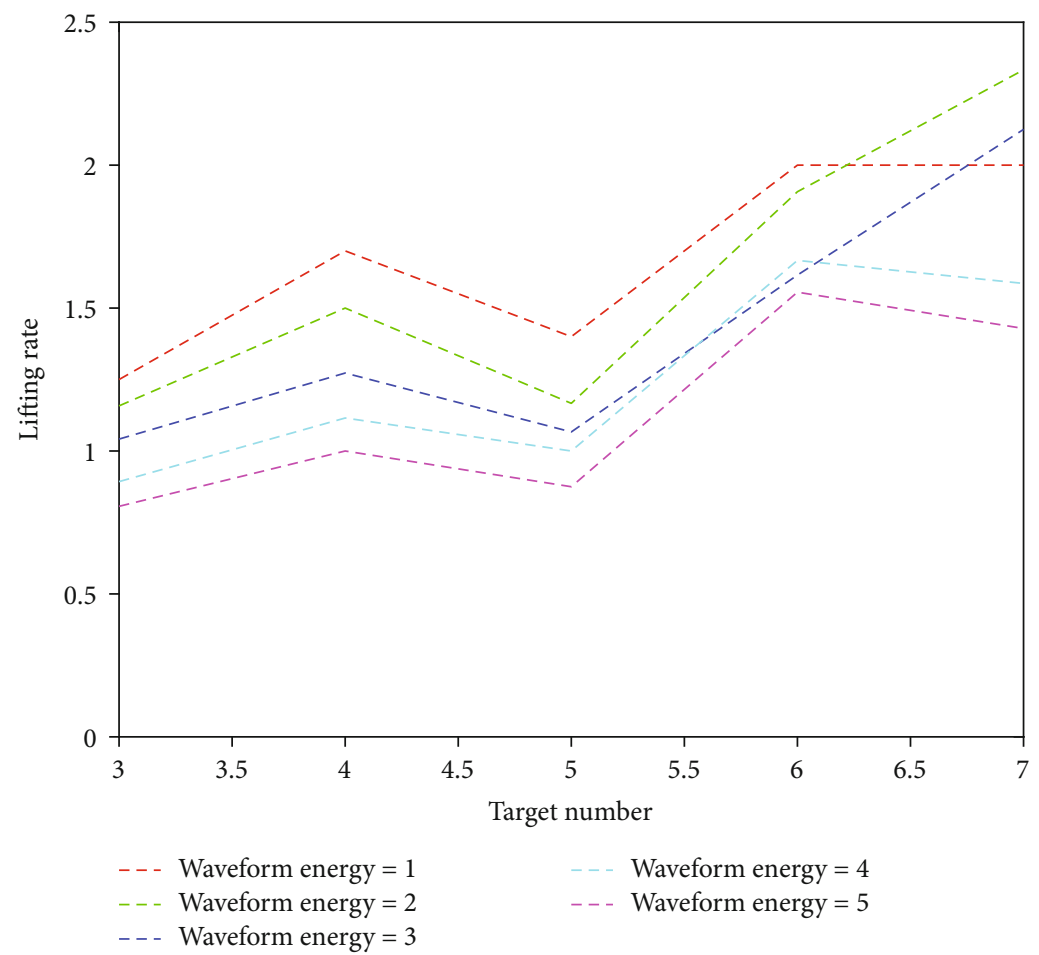

FIGURE 12: The relationship between the relative lifting rate and the number of targets in the situation of equal probability target.

abruptly at the abscissa of 4 , that is, when the target probabilities are not equal, the relative lifting rate of the target number greater than or equal to 4 is much better than the lifting rate of the target number less than 4 . In a word, in the situation of unequal probability, the robust waveform design based on harmonic variance is more suitable for the case when the number of targets is more than 4. In Figure 12, although the line has some fluctuations before the abscissa 5 , it starts to rise abruptly at abscissa 5 , that is, when the target probabilities are equal, the relative lifting rate of the target 
number greater than 5 is much better than that of the target less than or equal to 5 . In a word, in the situation of equal probability, the robust waveform design based on harmonic variance is more suitable for the case when the number of targets is more than 5 .

\section{Conclusion}

Adaptive waveform design is very important for cognitive radar. Generally, in the real scene, the targets detected by the radar are not known, and they are all random. We should consider the uncertainty of target and use robust technology for transmitted waveform. Therefore, on the basis of a variety of radar transmitted waveforms that have been studied, we set up a random target signal model based on MI and derive the optimal transmitted waveform. The real targets are random, so their emergence has certain uncertainty. The real target spectrum has certain uncertainty range. In order to make the worst case represented by the lower bound of the range more unfavorable, we introduce harmonic variance to make the lower bound smaller. Then we define the MI lifting rate of robust waveform based on harmonic variance relative to the optimal transmitted waveform in the uncertainty range. Simulation results show that, in the situation of multiple targets, compared to the original variance, the MI lifting rate of robust waveform based on harmonic variance relative to the optimal transmitted waveform in the uncertainty range has great improvement. Under the same conditions as the optimal transmitted waveform in the uncertain range, the more the number of targets is, the better performance the robust waveform based on harmonic variance is. In the situation of unequal probability, the robust waveform design based on harmonic variance is more suitable for the case when the number of targets is more than 4 . In the situation of equal probability, the robust waveform design based on harmonic variance is more suitable for the case when the number of targets is more than 5. In a word, robust waveform based on harmonic variance and $\mathrm{MI}$ is more suitable for more targets.

\section{Data Availability}

All data used to support the findings of this study are included within the article.

\section{Conflicts of Interest}

The authors declare that there are no conflicts of interest regarding the publication of this paper.

\section{Acknowledgments}

This work was supported by the Natural Science Foundation of Hebei Province (No. F2018501051).

\section{References}

[1] X. Cheng, A. Aubry, D. Ciuonzo, A. De Maio, and X. Wang, "Robust waveform and filter Bank Design of Polarimetric
Radar," IEEE Transactions on Aerospace and Electronic Systems, vol. 53, no. 1, pp. 370-384, 2017.

[2] Z. Zhu, S. Kay, and R. S. Raghavan, "Information-theoretic optimal radar waveform design," IEEE Signal Processing Letters, vol. 24, no. 3, pp. 274-278, 2017.

[3] L. Wang, P. V. Brennan, H. Wang, and K. K. Wong, "Minimax robust jamming techniques based on signal-to-interferenceplus-noise ratio and mutual information criteria," IET Communications, vol. 8, no. 10, pp. 1859-1867, 2014.

[4] W. Yuxi, H. Guoce, and L. Wei, "Waveform design for radar and extended target in the environment of electronic warfare," Journal of Systems Engineering and Electronics, vol. 29, no. 1, pp. 48-57, 2018.

[5] B. Wang, J. Wang, X. Song, and Y. Han, "A new waveform design method for cognitive radar," in 2009 Third International Symposium on Intelligent Information Technology Application, pp. 176-179, Shanghai, China, November 2009.

[6] L. Wang, W. Zhu, Y. Zhang, Q. Liao, and J. Tang, "Multi-target detection and adaptive waveform Design for Cognitive MIMO radar," IEEE Sensors Journal, vol. 18, no. 24, pp. 9962-9970, 2018.

[7] H.-S. Kim, N. A. Goodman, C. K. Lee, and S. I. Yang, "Improved waveform design for radar target classification," Electronics Letters, vol. 53, no. 13, pp. 879-881, 2017.

[8] C. Shi, F. Wang, M. Sellathurai, J. Zhou, and S. Salous, "Power minimization-based robust OFDM radar waveform design for radar and communication systems in coexistence," IEEE Transactions on Signal Processing, vol. 66, no. 5, pp. 13161330, 2018.

[9] B. Wang, X. Chen, S. Li, F. Xin, and J. Wang, "Robust waveform design based on jammer games in cognitive radar," in 2018 10th International Conference on Communications, Circuits and Systems (ICCCAS), pp. 195-198, Chengdu, China, December 2018.

[10] Y. Yao, P. Miao, and Z. Chen, "Cognitive waveform optimization for phase-modulation-based joint radar-communications system," IEEE Access, vol. 8, pp. 33276-33288, 2020.

[11] A. Leshem, O. Naparstek, and A. Nehorai, "Information theoretic adaptive radar waveform design for multiple extended targets," IEEE Journal of Selected Topics in Signal Processing, vol. 1, no. 1, pp. 42-55, 2007.

[12] D. Guo, S. Shamai, and S. Verdu, "Mutual information and minimum mean-square error in Gaussian channels," IEEE Transactions on Information Theory, vol. 51, no. 4, pp. 12611282, 2005.

[13] M. R. Bell, "Information theory and radar waveform design," IEEE Transactions on Information Theory, vol. 39, no. 5, pp. 1578-1597, 1993.

[14] J. Bae and N. A. Goodman, "Target recognition with highfidelity target signatures and adaptive waveforms in MIMO radar," in 2015 IEEE 6th International Workshop on Computational Advances in Multi-Sensor Adaptive Processing (CAMSAP), pp. 285-288, Cancun, Mexico, December 2015.

[15] C. Shi, F. Wang, M. Sellathurai, and J. Zhou, "Low probability of intercept-based distributed MIMO radar waveform design against barrage jamming in signal-dependent clutter and coloured noise," IET Signal Processing, vol. 13, no. 4, pp. 415-423, 2019. 